\title{
A finite-element approach to dynamical diffraction problems in reflection geometry
}

\section{Honkanen, Ari-Pekka}

2018-04

Honkanen, A-P , Ferrero , C , Guigay , J-P \& Mocella , V 2018 , ' A finite-element approach to dynamical diffraction problems in reflection geometry ' , Journal of Applied Crystallography , vol. 51 , pp. 514-525 . https://doi.org/10.1107/S1600576718001930

http://hdl.handle.net/10138/234485

https://doi.org/10.1107/S1600576718001930

Downloaded from Helda, University of Helsinki institutional repository.

This is an electronic reprint of the original article.

This reprint may differ from the original in pagination and typographic detail.

Please cite the original version. 


\title{
A finite-element approach to dynamical diffraction problems in reflection geometry
}

\author{
Ari-Pekka Honkanen, Claudio Ferrero, Jean-Pierre Guigay and Vito \\ Mocella
}

J. Appl. Cryst. (2018). 51, 514-525

\section{- IUCr Journals \\ CRYSTALLOGRAPHY JOURNALS ONLINE}

Copyright (C) International Union of Crystallography

Author(s) of this paper may load this reprint on their own web site or institutional repository provided that this cover page is retained. Republication of this article or its storage in electronic databases other than as specified above is not permitted without prior permission in writing from the IUCr.

For further information see http://journals.iucr.org/services/authorrights.html 


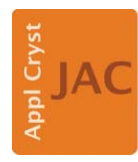

JOURNAL OF

APPLIED

CRYSTALLOGRAPHY

ISSN 1600-5767

Received 11 August 2017

Accepted 1 February 2018

Edited by S. Boutet, SLAC National Accelerator Laboratory, Menlo Park, USA

Keywords: finite-element approach; TakagiTaupin equations; dynamical diffraction; reflection geometry.

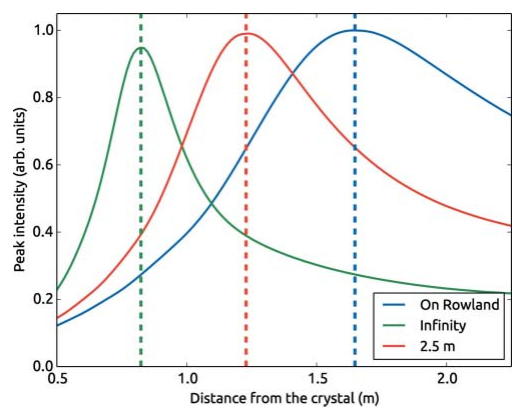

C 2018 International Union of Crystallography

\section{A finite-element approach to dynamical diffraction problems in reflection geometry}

\author{
Ari-Pekka Honkanen, ${ }^{a}$ Claudio Ferrero, ${ }^{b}$ Jean-Pierre Guigay ${ }^{b}$ and Vito Mocella ${ }^{\mathrm{c} *}$ \\ ${ }^{\mathbf{a}}$ Department of Physics, University of Helsinki, PO Box 64, FI-00014 Helsinki, Finland, ${ }^{\mathbf{b}}$ ESRF - The European \\ Synchrotron, Grenoble, France, and ${ }^{\mathrm{c} C N R-I M M}$ Sede di Napoli, via P. Castellino 111, I-80131 Napoli, Italy. \\ *Correspondence e-mail: vito.mocella@cnr.it
}

A finite-element approach to the numerical solution of the Takagi-Taupin equations expressed in a weak form is presented and applied to simulate the $\mathrm{X}$-ray reflectivity curves, spatial intensity distributions and focusing properties of bent perfect crystals in symmetric reflection geometry. The proposed framework encompasses a new formulation of the Takagi-Taupin equations, which appears to be promising in terms of robustness and stability and supports the Fresnel propagation of the diffracted waves. The presented method is very flexible and has the potential of dealing with dynamical X-ray or neutron diffraction problems related to crystals of arbitrary shape and deformation. The reference implementation based on the commercial COMSOL Multiphysics software package is available to the relevant user community.

\section{Introduction}

The Takagi-Taupin equations (TTE) are partial differential equations (PDEs) which describe the dynamical Bragg diffraction in a perfect or deformed crystal (Penning \& Polder, 1961; Takagi, 1962, 1969; Taupin, 1964; Authier, 2004; Apolloni et al., 2008). Analytical solutions exist for only a few cases of deformation (Katagawa \& Kato, 1974; Litzman \& Janácek, 1974; Chukhovskii et al., 1978). In general, one has to resort to numerical solution of the TTE. An approximate approach to solving the diffraction curves of large crystals was introduced recently (Honkanen et al., 2014, 2016), and an iterative method starting from an integral expression of the TTE and involving a series expansion was used by Yan \& $\mathrm{Li}$ (2014).

Traditionally, the TTE are solved (Authier et al., 1968; Balibar \& Authier, 1967; Epelboin \& Riglet, 1979; Epelboin, 1985; Gronkowski, 1991; Carvalho \& Epelboin, 1993a) using a finite difference (FD) scheme, easily implementable on a Cartesian mesh but not on an arbitrary (e.g. deformed) mesh. In principle, the FD scheme could be implemented on curved crystal surfaces using the reciprocity method (Carvalho \& Epelboin, 1993b), but this has yet to be done. Furthermore, the incident wave is usually considered to be either a plane wave with reference to an infinitely distant point source or a so-called 'spherical wave' with reference to a point source located on the crystal surface, whereas the intermediate case of an arbitrary finite distance between the source and the crystal applies to many actual situations (Lagomarsino et al., 2002).

Conversely, a finite-element method (FEM) based on a weak numerical form of the differential TTE can potentially deal very well with any kind of incident wave and crystals of any shape. A great advantage of this approach is that FEM implementations (Reddy, 2006; Oden \& Reddy, 2012) used for 
engineering problems are readily available and can be applied to X-ray diffraction problems (Mocella et al., 2003, 2015; Honkanen et al., 2017). One of the benefits of using the FEM is that it allows a great deal of flexibility in the selection of discretization, both in the elements that may be used to discretize space and in the so-called basis and test functions. Smaller elements in a region where the gradient of the soughtafter function is large could easily be used. Another considerable advantage of the FEM is that its theory is well established, owing to the close relationship between the numerical and the weak formulation of a PDE problem. In the present work, a FEM TTE solver is implemented in a commercial software package (COMSOL Multiphysics; http://www. comsol.com) and the method is verified in the case of Bragg reflection by perfect and cylindrically bent crystal plates. Bent crystals have frequently been used as focusing elements on $\mathrm{X}$-ray or neutron beamlines in both reflection and transmission geometry (e.g. Tolentino et al., 1988; Chukhovskii et al., 1994; Podorov et al., 2001; Mocella et al., 2004, 2008; Nesterets \& Wilkins, 2008; Sutter et al., 2010; Guigay \& Ferrero, 2016). The focusing properties of elliptical multilayers have also been studied (Guigay et al., 2008; Morawe et al., 2008; Osterhoff et al., 2013).

The structure of this paper is as follows. We will derive an alternative form of the TTE which is particularly suitable for the FEM at hand in terms of stability and computational efficiency. The boundary conditions for the derived TTE are discussed and set in place for reflection geometry. The propagation of the diffracted wavefield is examined in the context of Fresnel diffraction. The weak forms of the TTE are derived and the details of the COMSOL implementation are discussed. Finally, the validity of the method is investigated through a chosen set of simulations. This work is a further development of our previously published work (Honkanen et al., 2017).

\section{The Takagi-Taupin equations}

Let us consider a crystal in Bragg diffraction geometry in which the incident beam is represented by a $\sigma$-polarized (the $\pi$-polarization case can be described similarly) monochromatic modulated plane wave of the form

$$
\psi_{\text {inc }}(\mathbf{r})=E_{\text {inc }}(\mathbf{r}) \exp \left(i \mathbf{k}_{0} \cdot \mathbf{r}\right)
$$

The length of the wavevector $\mathbf{k}_{0}$ is $2 \pi / \lambda$, where $\lambda$ is the wavelength of the X-rays. The diffracted wave in air can be written analogously,

$$
\psi_{\text {out }}(\mathbf{r})=E_{\text {out }}(\mathbf{r}) \exp \left(i \mathbf{k}_{h} \cdot \mathbf{r}\right)
$$

where $\mathbf{k}_{h}=\mathbf{k}_{0}+\mathbf{h}$, with $\mathbf{h}$ being the reciprocal vector corresponding to the diffractive planes.

In a non-homogeneous medium, the wavefield $\psi$ fulfils the general wave equation

$$
\nabla^{2} \psi+k^{2}[1+\chi(\mathbf{r})] \psi=0
$$

of which the solution in the usual two-beam case is of the form

$$
\psi(\mathbf{r})=E_{0}(\mathbf{r}) \exp \left(i \mathbf{k}_{0} \cdot \mathbf{r}\right)+E_{h}(\mathbf{r}) \exp \left(i \mathbf{k}_{h} \cdot \mathbf{r}\right) .
$$

For a periodic deformed medium, the susceptibility $\chi$ can be expanded in a Fourier-series-like manner as follows:

$$
\chi(\mathbf{r})=\chi_{0}+\chi_{\bar{h}} \exp [-i \mathbf{h} \cdot(\mathbf{r}-\mathbf{u})]+\chi_{h} \exp [i \mathbf{h} \cdot(\mathbf{r}-\mathbf{u})]+\cdots,
$$

where $\mathbf{u}$ is the displacement field. By multiplying equations (5) and (4) and retaining only the terms relevant to the two-beam case, we obtain

$$
\begin{aligned}
\chi(\mathbf{r}) \psi(\mathbf{r}) \simeq & {\left[\chi_{0} E_{0}+\chi_{\bar{h}} \exp (i \mathbf{h} \cdot \mathbf{u}) E_{h}\right] \exp \left(i \mathbf{k}_{0} \cdot \mathbf{r}\right) } \\
& +\left[\chi_{0} E_{h}+\chi_{h} \exp (-i \mathbf{h} \cdot \mathbf{u}) E_{0}\right] \exp \left(i \mathbf{k}_{h} \cdot \mathbf{r}\right) .
\end{aligned}
$$

Since $E_{0, h}$ vary slowly compared with $\exp \left(i \mathbf{k}_{0, h} \cdot \mathbf{r}\right)$, their second-order derivatives arising in $\nabla^{2} \psi$ can be neglected. Hence the following approximation applies:

$$
\begin{aligned}
\nabla^{2} \psi \simeq & \left(2 i \mathbf{k}_{0} \cdot \nabla E_{0}-\mathbf{k}_{0}^{2} E_{0}\right) \exp \left(i \mathbf{k}_{0} \cdot \mathbf{r}\right) \\
& +\left(2 i \mathbf{k}_{h} \cdot \nabla E_{h}-\mathbf{k}_{h}^{2} E_{h}\right) \exp \left(i \mathbf{k}_{h} \cdot \mathbf{r}\right) .
\end{aligned}
$$

By substituting equations (6) and (7) into (3), we obtain

$$
\begin{gathered}
2 \mathbf{k}_{0} \cdot \nabla E_{0}=i\left[k^{2}\left(1+\chi_{0}\right)-k_{0}^{2}\right] E_{0}+i k^{2} \chi_{\bar{h}} E_{h} \exp (i \mathbf{h} \cdot \mathbf{u}), \\
2 \mathbf{k}_{h} \cdot \nabla E_{h}=i\left[k^{2}\left(1+\chi_{0}\right)-k_{h}^{2}\right] E_{h}+i k^{2} \chi_{h} E_{0} \exp (-i \mathbf{h} \cdot \mathbf{u}) .
\end{gathered}
$$

Equations $(8 a)$ and $(8 b)$ can be simplified by noting that $k_{0}^{2}=$ $k^{2}$ and $\left(k_{h}^{2}-k_{0}^{2}\right) / 2 k_{h} \simeq k-k_{h} \simeq k \Delta \theta \sin 2 \theta_{\mathrm{B}}$, where $\theta_{\mathrm{B}}$ is the Bragg angle and $\Delta \theta=\theta-\theta_{\mathrm{B}}, \theta$ being the glancing angle of the incident wavevector $\mathbf{k}_{0}$ on the diffracting Bragg planes.

It is convenient to consider $E_{0, h}(\mathbf{r})$ as the functions $E_{0, h}\left(s_{0}, s_{h}\right)$ of the oblique coordinates $s_{0}$ and $s_{h}$ along the directions of $\mathbf{k}_{0}$ and $\mathbf{k}_{h}$, respectively. As shown in Appendix $A$, for any function $F\left(s_{0}, s_{h}\right)$ with gradient $\nabla F$, it holds that $\mathbf{k}_{0, h} \cdot \nabla F=k_{0, h} \partial_{0, h} F\left(s_{0}, s_{h}\right)$, where $\partial_{0, h}$ denotes the partial derivative with respect to $s_{0, h}$. Thus, equations $(8 a)$ and $(8 b)$ become

$$
\begin{gathered}
2 \partial_{0} E_{0}=i k \chi_{0} E_{0}+i k \chi_{\bar{h}} E_{h} \exp (i \mathbf{h} \cdot \mathbf{u}), \\
2 \partial_{h} E_{h}=i\left[k \chi_{0}+2\left(k-k_{h}\right)\right] E_{h}+i k \chi_{h} E_{0} \exp (-i \mathbf{h} \cdot \mathbf{u}) .
\end{gathered}
$$

The case of $\pi$-polarization can be included in this formalism by replacing the coefficients $\chi_{\bar{h}, h}$ by $C \chi_{\bar{h}, h}$, where $C=1$ or $\cos 2 \theta_{\mathrm{B}}$ for $\sigma$ - and $\pi$-polarization, respectively. By using the notation $c_{0}=k \chi_{0} / 2, c_{\bar{h}, h}=k C \chi_{\bar{h}, h} / 2, \beta=k-k_{h} \simeq k \Delta \theta \sin 2 \theta_{\mathrm{B}}$ and introducing the functions

$$
\begin{gathered}
D_{0}\left(s_{0}, s_{h}\right)=E_{0}\left(s_{0}, s_{h}\right) \exp \left(-i k \chi_{0} \frac{s_{0}+s_{h}}{2}\right), \\
D_{h}\left(s_{0}, s_{h}\right)=E_{h}\left(s_{0}, s_{h}\right) \exp \left[-i k \chi_{0} \frac{s_{0}+s_{h}}{2}+i \mathbf{h} \cdot \mathbf{u}\left(s_{0}, s_{h}\right)\right],
\end{gathered}
$$

equations $(9 a)$ and $(9 b)$ can be written as

$$
\begin{gathered}
\partial_{0} D_{0}=i c_{\bar{h}} D_{h}, \\
\partial_{h} D_{h}=i\left[\beta+\partial_{h}(\mathbf{h} \cdot \mathbf{u})\right] D_{h}+i c_{h} D_{0} .
\end{gathered}
$$


Equations (12a) and (12b) are the most usual form of the TTE. However, for the reasons explained in the next section, it is more convenient to use a modified expression in terms of the functions

$$
\begin{gathered}
\Gamma_{0}=E_{0} \exp \left(-i \beta s_{h}\right), \\
\Gamma_{h}=E_{h} \exp \left(-i \beta s_{h}+i \mathbf{h} \cdot \mathbf{u}\right) .
\end{gathered}
$$

By substituting the above equations into $(9 a)$ and $(9 b)$, the TTE become

$$
\begin{gathered}
\partial_{0} \Gamma_{0}=i c_{0} \Gamma_{0}+i c_{\bar{h}} \Gamma_{h}, \\
\partial_{h} \Gamma_{h}=i\left[c_{0}+\partial_{h}(\mathbf{h} \cdot \mathbf{u})\right] \Gamma_{h}+i c_{h} \Gamma_{0} .
\end{gathered}
$$

Equations (15a) and (15b) form the basis of our FEM implementation. The main advantage gained by moving the $\beta$ term out of the equation to the boundary conditions is the increased stability. The reason behind this can be understood by considering equation (12b). At the large $\beta$ limit, the solution of $D_{h}$ is found to be proportional to $\exp \left(i \beta s_{h}\right)$, meaning that the phase of the solution oscillates rapidly along the propagation direction of the diffracted beam. On the length scale of the problem, these oscillations even out and thus have little physical consequence. However, they cause a major computational difficulty. This problem is avoided by moving $\beta$ to the surface term, as $s_{h}$ varies more slowly along the surface than it would along the direction of the diffracted beam path. Thus, a sparser solving grid can be used, leading to shorter computation times and less heavy memory usage.

\section{Boundary conditions for the reflection geometry}

The handling of the TTE in $\$ 2$ is valid for reflection, transmission and mixed cases. The different cases are separated from each other via the boundary conditions. For simplicity, we focus solely on the reflection geometry henceforth.

On the entrance surface of the incident wave, the boundary condition for $\Gamma_{0}$ is given by

$$
\Gamma_{0}\left(\mathbf{r}_{\text {surf }}\right)=E_{0}\left(\mathbf{r}_{\text {surf }}\right) \exp \left(-i \beta s_{h, \text { surf }}\right),
$$

where $s_{h \text {,surf }}=s_{h}\left(\mathbf{r}_{\text {surf }}\right)$ is subject to the choice of the origin. For instance, for an incident plane wave $\psi_{\text {inc,plane }}=E_{0} \exp \left(i \mathbf{k}_{0} \cdot \mathbf{r}\right)$

$$
\Gamma_{0, \text { plane }}\left(\mathbf{r}_{\text {surf }}\right)=E_{0} \exp \left(-i \beta s_{h, \text { surf }}\right),
$$

where $E_{0}$ is constant in this case. On the other hand, for a divergent source

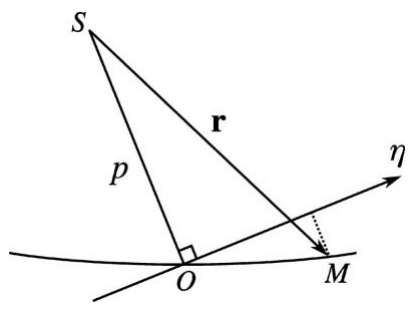

Figure 1

The nomenclature used in the paraxial approximation.

$$
\psi_{\text {inc, div }}=\frac{A}{r^{\gamma}} \exp (i k r)
$$

where $A$ is a constant and $\gamma=1 / 2$ for a line source and 1 for point source. By denoting $\mathbf{r}=\overline{S M}$, the position vector of a point $M$ on the crystal surface with respect to the source $S$, and with $\eta$ being the coordinate perpendicular to $\mathbf{k}_{0}$ (see Fig. 1), we may use the so-called paraxial approximation

$$
k r-\mathbf{k}_{0} \cdot \mathbf{r} \simeq \frac{k \eta^{2}}{2 r} \simeq \frac{k \eta^{2}}{2 p},
$$

where $p$ is the distance from $S$ to the origin $O$ on the crystal surface such that the ray $\overline{S O}$ corresponds to the exact incident Bragg direction.

We thus obtain $\psi_{\text {inc,div }} \simeq A p^{-\gamma} \exp \left(i \mathbf{k}_{0} \cdot \mathbf{r}+i k \eta^{2} / 2 p\right)$, so the boundary condition becomes

$$
\Gamma_{0, \text { div }}\left(\mathbf{r}_{\text {surf }}\right)=A p^{-\gamma} \exp \left(i k \frac{\eta^{2}}{2 p}-i \beta s_{h}\right) .
$$

In addition, the boundary condition for $\Gamma_{0}$ is left free (i.e. to be solved) on the exit surface of the incident wave and set to zero elsewhere (Fig. 2).

For the diffracted wave $\Gamma_{h}$, the boundary condition is $\Gamma_{h}=0$ everywhere else, except on the exit surface where it is left free. Note that the different surfaces may overlap with each other. The different boundaries are illustrated in Fig. 2.

\section{Propagation of the diffracted wave}

In order to describe the propagation of the reflected beam in air, we use the rectangular coordinates $(\xi, q)$ as depicted in Fig. 3. For the solved $\Gamma_{h}$, the diffracted wave on the crystal surface is obtained by

$$
\psi_{\text {out }}\left(\mathbf{r}_{\text {surf }}\right)=\Gamma_{h}\left(\mathbf{r}_{\text {surf }}\right) \exp \left(i \mathbf{k}_{h} \cdot \mathbf{r}_{\text {surf }}+i \beta s_{h, \text { surf }}-i \mathbf{h} \cdot \mathbf{u}_{\text {surf }}\right),
$$

with $s_{h \text {,surf }}=s_{h}\left(\mathbf{r}_{\text {surf }}\right)$ and $\mathbf{u}_{\text {surf }}=\mathbf{u}\left(\mathbf{r}_{\text {surf }}\right)$. Since the diffracted wave is in essence a modulated plane wave, we can propagate it (in the mathematical sense) in the vicinity of the surface simply by adjusting its phase by $\exp \left(i k \Delta s_{h}\right)$.

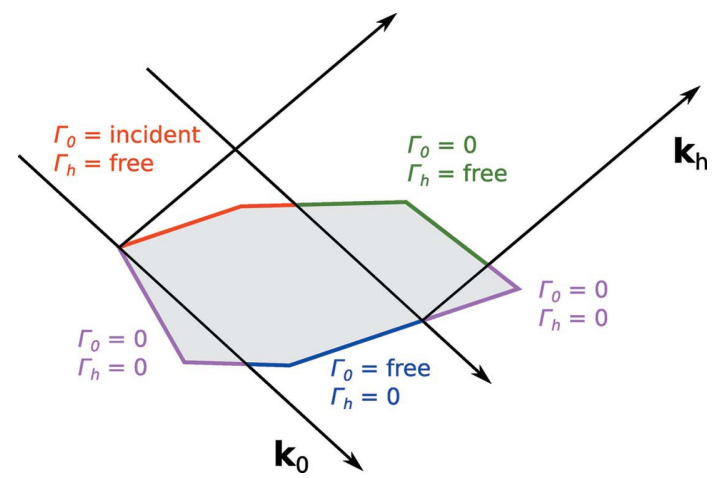

Figure 2

Boundary conditions for the reflection geometry. Red denotes the entrance surface of the incident wave, blue the exit surface of the incident wave, red + green the exit surface of the diffracted wave and magenta the boundaries outside the domain of diffraction. 
Now, let us consider the plane which goes through the origin $\left(s_{h}=0\right)$ and is perpendicular to $s_{h}$. If we propagate $\psi_{\text {out }}$ from the crystal surface on this plane, as indicated in Fig. 3, we find that

$$
\psi_{\text {out }, \text { plane }}(\xi)=\Gamma_{h}\left[\mathbf{r}_{\text {surf }}(\xi)\right] \exp \left[i \beta s_{h, \text { surf }}(\xi)-i \mathbf{h} \cdot \mathbf{u}_{\text {surf }}(\xi)\right],
$$

where $\mathbf{r}_{\text {surf }}(\xi), s_{h, \text { surf }}(\xi)$ and $\mathbf{u}_{\text {surf }}(\xi)$ are evaluated at $\mathbf{r}_{\text {surf }}$ with the same $\xi$ coordinate.

Equation (22) allows us to use the Fresnel diffraction integral to compute the wave amplitude far away from the crystal. In a plane at a distance $q$ from the origin, the wave amplitude is

$$
\Psi(\xi, q)=\frac{1}{(\lambda q)^{1 / 2}} \int \psi_{\text {out,plane }}\left(\xi^{\prime}\right) \exp \left[\frac{i k\left(\xi-\xi^{\prime}\right)^{2}}{2 q}\right] \mathrm{d} \xi^{\prime} .
$$

\section{Weak formulation of Takagi-Taupin equations}

Following the well established FEM procedure, both sides of equations $(15 a)$ and $(15 b)$ are multiplied by test functions $v_{1}\left(s_{0}, s_{h}\right)$ and $v_{2}\left(s_{0}, s_{h}\right)$ and integrated over the domain $\Omega$ with the boundary $\delta \Omega$ :

$$
\begin{gathered}
\int_{\Omega}\left(\partial_{0} \Gamma_{0}-i c_{0} \Gamma_{0}-i c_{\bar{h}} \Gamma_{h}\right) v_{1} \mathrm{~d} V=0, \\
\int_{\Omega}\left\{\partial_{h} \Gamma_{h}-i\left[c_{0}+\partial_{h}(\mathbf{h} \cdot \mathbf{u})\right] \Gamma_{h}-i c_{h} \Gamma_{0}\right\} v_{2} \mathrm{~d} V=0 .
\end{gathered}
$$

Let $\mathbf{s}_{0}$ and $\mathbf{s}_{h}$ be the unit vectors along the directions of $\mathbf{k}_{0}$ and $\mathbf{k}_{h}$, respectively. According to Appendix $A$, we can write

$$
\begin{aligned}
& v_{1} \partial_{0} \Gamma_{0}=\partial_{0}\left(v_{1} \Gamma_{0}\right)-\Gamma_{0} \partial_{0} v_{1}=\nabla \cdot\left(v_{1} \Gamma_{0} \mathbf{s}_{0}\right)-\Gamma_{0} \partial_{0} v_{1}, \\
& v_{2} \partial_{h} \Gamma_{h}=\partial_{h}\left(v_{2} \Gamma_{h}\right)-\Gamma_{h} \partial_{h} v_{2}=\nabla \cdot\left(v_{2} \Gamma_{h} \mathbf{s}_{h}\right)-\Gamma_{h} \partial_{h} v_{2} .
\end{aligned}
$$

Utilizing the divergence theorem, we can transform the volume integrals over the divergence terms into the following surface integrals:

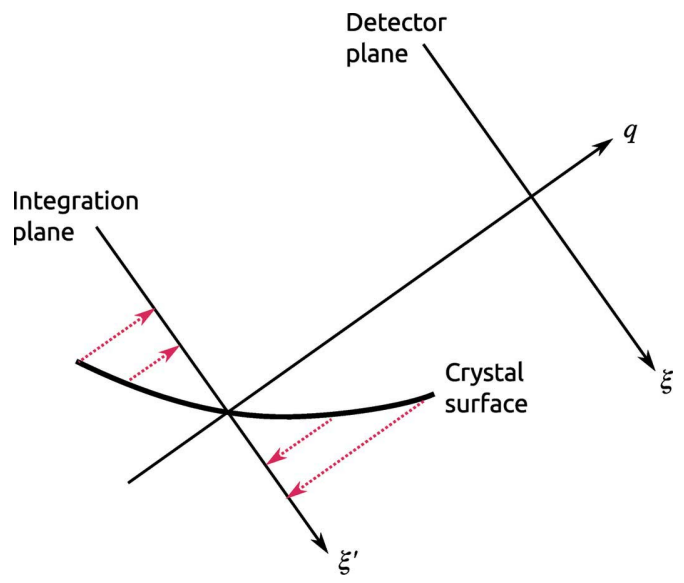

Figure 3

The auxiliary planes and coordinate system for computing the wave propagation. In the vicinity of the crystal, the diffracted wave is propagated on the integration plane as a plane wave, from which it is further propagated to the detector plane using the Fresnel diffraction integral.

$$
\begin{aligned}
& \int_{\Omega} \nabla \cdot\left(v_{1} \Gamma_{0} \mathbf{s}_{0}\right) \mathrm{d} V=\int_{\delta \Omega} v_{1} \Gamma_{0} \mathbf{s}_{0} \cdot \mathbf{n} \mathrm{d} S, \\
& \int_{\Omega} \nabla \cdot\left(v_{2} \Gamma_{h} \mathbf{s}_{h}\right) \mathrm{d} V=\int_{\delta \Omega} v_{2} \Gamma_{h} \mathbf{s}_{h} \cdot \mathbf{n} \mathrm{d} S,
\end{aligned}
$$

where $\mathbf{n}$ is the unit outward normal on $\delta \Omega$. Thus, we finally obtain

$$
\int_{\Omega}\left(\Gamma_{0} \partial_{0} v_{1}+i c_{0} v_{1} \Gamma_{0}+i c_{\bar{h}} v_{1} \Gamma_{h}\right) \mathrm{d} V-\int_{\delta \Omega} v_{1} \Gamma_{0} \mathbf{s}_{0} \cdot \mathbf{n} \mathrm{d} S=0,
$$

$$
\int_{\Omega}\left(\Gamma_{h} \partial_{h} v_{2}+i c_{0}^{\prime} v_{2} \Gamma_{h}+i c_{h} v_{2} \Gamma_{0}\right) \mathrm{d} V-\int_{\delta \Omega} v_{2} \Gamma_{h} \mathbf{s}_{h} \cdot \mathbf{n} \mathrm{d} S=0,
$$

where $c_{0}^{\prime}=c_{0}+\partial_{h}(\mathbf{h} \cdot \mathbf{u})$.

Equations (29a) and (29b) represent the so-called 'weak' or 'variational' formulation of the differential equations (15a) and $(15 b)$. The test functions $v_{1}$ and $v_{2}$ as well as $\Gamma_{0}$ and $\Gamma_{h}$ are assumed to belong to an infinite-dimensional Hilbert space $H$. It is required that these equalities hold for all test functions in $H$. In practice, however, the application of the FEM on these functions converts them to functions in a finite-dimensional function space and then to ordinary Euclidean vectors (in a vector space) that can be managed via numerical methods.

This formulation is called 'weak' because it relaxes the requirement expressed by (15a) and (15b), where all the terms of the PDEs must be defined at each point (point-wise formulation). The relations in (29a) and (29b), instead, only entail equality in an integral sense. As an example, a discontinuity in the first derivative of the solution function does not preclude integration. It introduces, however, a distribution (in a mathematical sense) for the second derivative. Note that, in such a case, equations (15a) and (15b) become immaterial in a discontinuity point.

In contrast with (24a) and (24b), equations (29a) and (29b) do not contain derivatives of the functions $\Gamma_{0}$ and $\Gamma_{h}$. They can be implemented in a FEM code, using a mesh of two-dimensional elements (often triangles, but rectangles or even higherorder elements are also used) adapted to the crystal shape in quite a straightforward fashion.

The solutions of $(29 a)$ and $(29 b)$ are expressed as $\Gamma_{0}(\mathbf{r})=$ $\sum_{i} \Gamma_{0, i} N_{i}(\mathbf{r})$ and $\Gamma_{h}(\mathbf{r})=\sum_{i} \Gamma_{h, i} N_{i}(\mathbf{r})$, respectively, where the sums go over all $n$ knots in the mesh, and $\Gamma_{0, i}$ and $\Gamma_{h, i}$ are coefficients to be determined. $N_{i}(\mathbf{r})$ are the basis (or shape) functions related to the $i$ th knot. Basis functions are nonzero everywhere, except in the vicinity of the knot they are tied to. Customarily, they are polynomial functions (e.g. B-splines) of degree one or higher; in this work quadratic functions were used. The well known Galerkin method (used also in this work) uses a set of test functions identical to the basis functions, i.e. $v_{1, j}(\mathbf{r})=v_{2, j}(\mathbf{r})=N_{j}(\mathbf{r})$. By transforming equations (15a) and (15b) into their weak form, the problem of solving this pair of PDEs is then reduced to solving a system of $2 n$ algebraic linear equations from which the coefficients $\Gamma_{0, i}$ and $\Gamma_{h, i}$ are to be determined numerically.

One of the most outstanding assets of the FEM is its ability to choose test and basis functions among a wide host of 
functions. It is often beneficial to select test and basis functions with a locally variable geometric support. It should be remembered that not all the highlighted features reported above are present in the FD approach, thus potentially making the FD solution of the same problem considerably more laborious and less efficient than the analogous FEM solution.

\section{Notes on the reference implementation}

The method was implemented in the commercial modelling and simulation software COMSOL Multiphysics 5.3. COMSOL Multiphysics was chosen owing to its widespread use and its readily available structural mechanics and heatconduction modules that can be used to solve the deformation field for TTE computation in the future development of the method.

The method was implemented using the Weak Form PDE interface, which makes it easy for the user to include arbitrary weakly formulated differential equations into the system. In addition to the strain component included in the TTE, the deformation was taken into account by including the displacement vector field $\mathbf{u}$ into the mesh geometry through the Moving Mesh interface.

Meshing of the crystal domain was done using the Free Triangular node, which automatically generates an unstructured mesh grid of triangular elements according to the given limitations on element sizes etc. The grid parameter of most relevance to this work was the Maximum element size, which limits the maximum distance of the grid nodes. For a simple rectangular geometry, this value corresponds to the typical node separation inside the domain. Later in this discussion we refer to this parameter simply as the (grid) element size.

As Multiphysics uses a Cartesian coordinate system, the oblique coordinates $\left(s_{0}, s_{h}\right)$ need to be transformed into Cartesian ones. The relation between the two systems is presented in Fig. 4. The unit vectors $\mathbf{s}_{0}$ and $\mathbf{s}_{h}$ in the Cartesian basis $\left\{\mathbf{e}_{x}, \mathbf{e}_{y}\right\}$ are

$$
\mathbf{s}_{0}=\cos \alpha \mathbf{e}_{x}-\sin \alpha \mathbf{e}_{y}, \quad \mathbf{s}_{h}=\cos \alpha^{\prime} \mathbf{e}_{x}+\sin \alpha^{\prime} \mathbf{e}_{y} .
$$

Thus, the oblique coordinates in terms of $x$ and $y$ are

$$
s_{0}=\frac{x \sin \alpha^{\prime}-y \cos \alpha^{\prime}}{\sin \left(\alpha+\alpha^{\prime}\right)}, \quad s_{h}=\frac{x \sin \alpha+y \cos \alpha}{\sin \left(\alpha+\alpha^{\prime}\right)},
$$

and the partial derivatives are

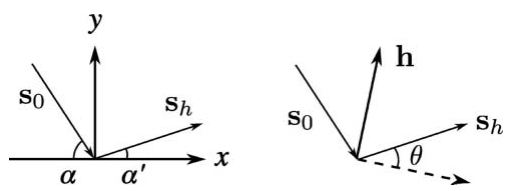

Figure 4

Direction vectors $\mathbf{s}_{0}$ and $\mathbf{s}_{h}$ of the incident and diffracted waves with respect to the Cartesian coordinate system $(x, y)$. The sign convention is so that both angles are positive for the case depicted. Note that the coordinate system is not, in the general case, aligned with $\mathbf{h}$.
Table 1

Crystal parameters and diffraction-related quantities for symmetric $\mathrm{Si}(111)$ at a photon energy of $6 \mathrm{keV}(\sigma$-polarized).

\begin{tabular}{ll}
\hline$\chi_{0}$ & $-0.274564 \times 10^{-4}+i 0.109657 \times 10^{-5}$ \\
$\chi_{h}$ & $-0.109980 \times 10^{-4}-i 0.991441 \times 10^{-5}$ \\
$\chi_{\bar{h}}$ & $-0.991441 \times 10^{-5}+i 0.109980 \times 10^{-4}$ \\
Bragg angle $\theta_{\mathrm{B}}$ & $19.24^{\circ}$ \\
Interplanar distance & $3.14 \AA$ \\
Absorption length & $29.99 \mu \mathrm{m}$ \\
Darwin width & $9.83^{\prime \prime}(47.7 \mu \mathrm{rad})$ \\
Extinction depth & $0.73 \mu \mathrm{m}$ \\
Extinction length & $2.22 \mu \mathrm{m}$ \\
Refraction correction & $9.12^{\prime \prime}(44.2 \mu \mathrm{rad})$
\end{tabular}

$$
\frac{\partial}{\partial s_{0}}=\cos \alpha \frac{\partial}{\partial x}-\sin \alpha \frac{\partial}{\partial y}, \quad \frac{\partial}{\partial s_{h}}=\cos \alpha^{\prime} \frac{\partial}{\partial x}+\sin \alpha^{\prime} \frac{\partial}{\partial y} \text {. }
$$

For the propagation calculations, the solved complex wave amplitudes on the crystal surface are exported into a text file. A program written in Python is used to read the contents of the files and compute the Fresnel integral for all points on the detector plane.

\section{Simulations}

In order to validate our FEM method, we solved the TTE for the symmetric $\mathrm{Si}(111)$ reflection for undeformed and cylindrically bent crystals for various incidence angles. The energy of the $\sigma$-polarized incident X-rays was set to $E=6 \mathrm{keV}$. The $\chi_{0, h, \bar{h}}$ values, together with diffraction-related quantities, were computed with XOP 2.4 (Sanchez del Rio \& Dejus, 2011; Sanchez del Rio et al., 2015) and are presented in Table 1.

\subsection{Reflectivity curves of the undeformed crystal}

The TTE were solved for various incidence angles for an undeformed rectangular crystal slab. The thickness $t$ of the crystal was set to $50 \mu \mathrm{m}$. In order to avoid disturbances caused by the sides of the crystal, the incident plane wave of equation (16) was multiplied by a Gaussian window function. The full width at half-maximum (FWHM) of the window was chosen to be $100 \mu \mathrm{m}$. Note that this FWHM applies for the amplitude of the wave; for the intensity of the wave, the given value should be regarded as the full width at a quarter of the maximum (FWQM). The width of the crystal was chosen to be $200 \mu \mathrm{m}$, which accommodates the masked beam well. For the aforementioned parameters, the simulated crystal can be considered thick in terms of the diffraction.

The reflectivity or rocking curves (RCs) were solved using maximum triangular element sizes of $0.5,1,1.75$ and $2.5 \mu \mathrm{m}$ (Fig. 5). As expected, the result converges towards the reference curve computed with $X O P$ as the element size gets smaller. The largest effect of the grid density can be seen on the top of the curve. This is natural, since this is the region of the RC where the dominating length scale is the extinction length. Compared with the extinction length of $2.22 \mu \mathrm{m}$, we find that the grid size needs to be two or three times smaller in order to obtain satisfactory convergence. 
The deviations seen in the tails of the RCs for the 1.75 and $2.5 \mu \mathrm{m}$ grids arise from the oscillating phase factor $\exp \left(-i \beta s_{h}\right)$

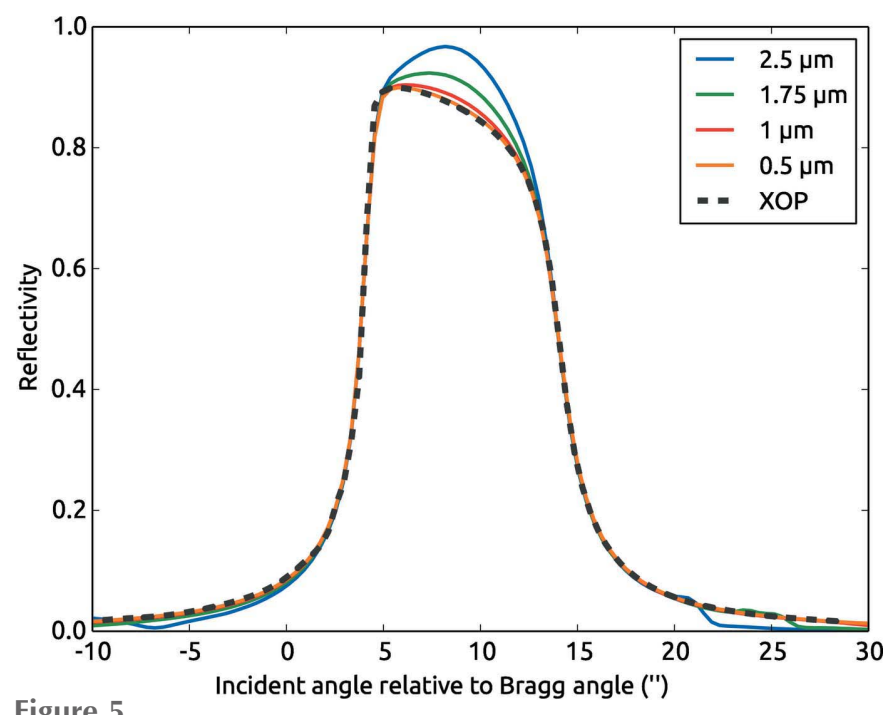

Figure 5

The reflectivity curves of the simulated undeformed $\mathrm{Si}(111)$ crystal, computed with different grid element sizes. The reference curve is obtained using $X I N P R O$ in $X O P$.

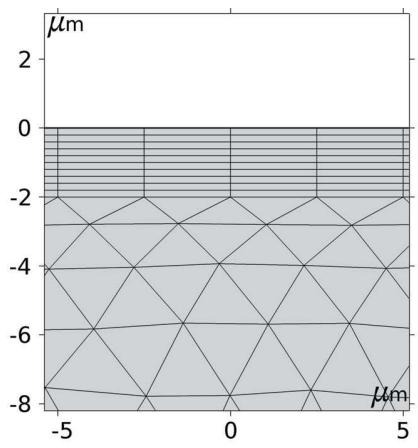

(a)

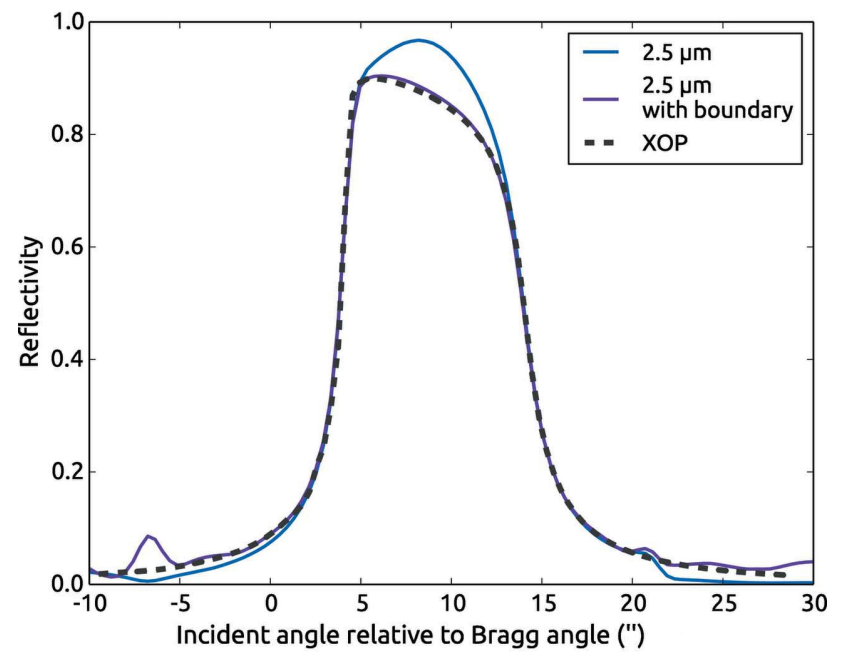

(b)

Figure 6

(a) The dense boundary layer on the surface. The number of rows of elements was ten and the separation between rows was $0.2 \mu \mathrm{m}$. (b) The reflectivity curves computed on a $2.5 \mu \mathrm{m}$ grid with and without the boundary layer. in the boundary condition of $\Gamma_{0}$. Using Bragg's law, the phase factor on the top surface can be written as

$$
\begin{aligned}
\exp \left(-i \beta s_{h}\right) & =\exp \left(-i k \Delta \theta \sin 2 \theta_{\mathrm{B}} \frac{x}{2 \cos \theta_{\mathrm{B}}}\right) \\
& =\exp \left(-2 \pi i \frac{\Delta \theta}{2 d} x\right)
\end{aligned}
$$

where $d$ is the interplanar distance of the Bragg planes. According to the Nyquist-Shannon sampling theorem, in order to sample a function at a frequency of $\Delta \theta / 2 d$, the separation of grid points $\Delta x$ needs to be $\leq d /|\Delta \theta|$. For $d=$ $3.14 \AA$ and $\Delta \theta=30^{\prime \prime}(145.4 \mu \mathrm{rad})$, we find that $\Delta x \leq 2.2 \mu \mathrm{m}$, which is in accordance with the simulations.

A natural choice within the FEM framework would be to modify the mesh density locally, following the variations in length scale of the solution. For the problem at hand, the shorter length-scale variation (extinction length) takes place near the surface. Using the Boundary Layer option available in Multiphysics, one can increase the density of the mesh near the surface and thus improve the accuracy of the RCs (Fig. 6). However, the introduction of a boundary layer causes some deviations in the tails of the RCs, calling for a deeper investigation of the meshing process, which is outside the scope of the present work.

\subsection{Reflectivity curves of cylindrically bent crystals}

The effect of cylindrical bending on reflectivity properties was investigated by including the bending field presented in Appendix $B$. Using the Poisson ratio $v=0.27$ and the crystal parameters tabulated in Table 1, the cylindrical bending can be considered weak when the bending radius $R \gg 0.12 \mathrm{~m}$, according to equation (46).

To examine the weakly deformed case, we set $R=5 \mathrm{~m}$. The crystal thickness was $50 \mu \mathrm{m}$, as before, but the width was extended by adding $200 \mu \mathrm{m}$ to the right-hand side (totalling $500 \mu \mathrm{m})$ in order to accommodate the curved beam path. A line-source boundary condition [equation (20)] multiplied by a Gaussian window (FWHM of amplitude $=100 \mu \mathrm{m}$ ) was used for the incident wave. A grid element size of $0.75 \mu \mathrm{m}$ was used.

The RCs for various source distances are presented in Fig. 7. When the line source is on the Rowland circle $(p=1.648 \mathrm{~m})$, the RC deviates only a little from the undeformed reference curve, which is an expected result for a weakly deformed crystal. The slight shift of the curve to the left can be associated with a decreased lattice spacing in the upper part of the crystal owing to the bending and the nonzero Poisson ratio. A part of the added weight on the left-hand side can also be explained by the so-called mirage effect (Gronkowski \& Malgrange, 1984; Gronkowski, 1991; Authier, 2004), which can be seen in Fig. 8, where the total intensity inside the crystal at $\Delta \theta=1.31^{\prime \prime}(6.37 \mu \mathrm{rad})$ is visualized. The incident cylindrical wave, which is approximately a plane wave with the incidence angle outside the Darwin range, excites a wavefield deep in the crystal owing to the locally changing orientation of the reflecting planes along the beam trajectory in the crystal. 


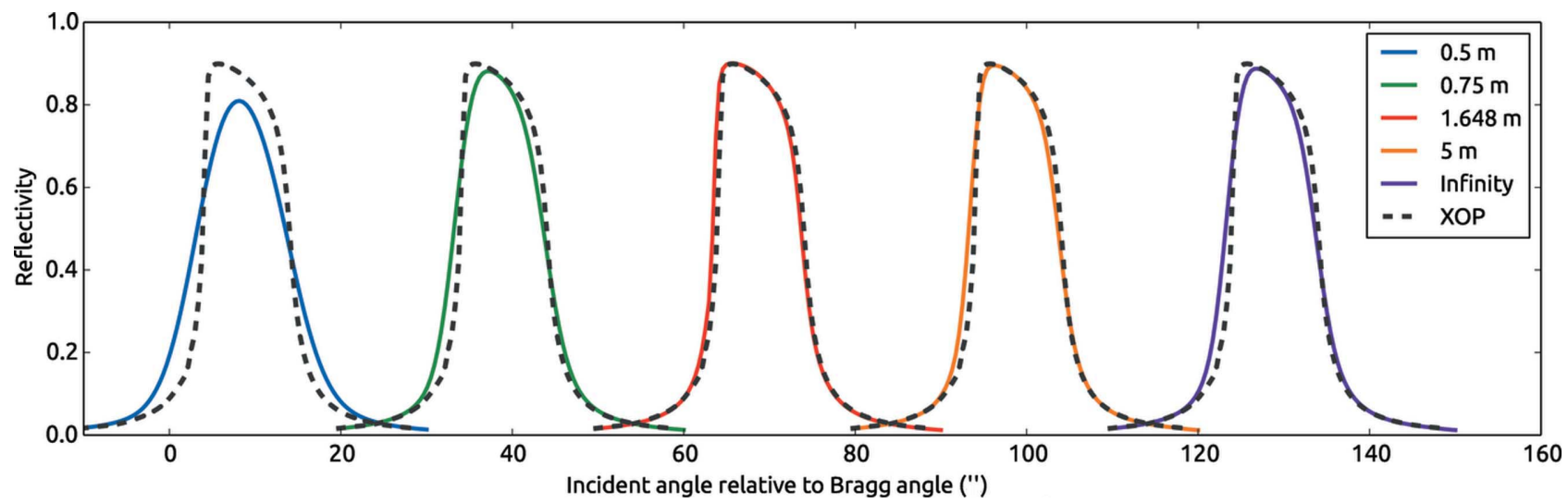

Figure 7

The reflectivity curves of the simulated cylindrically bent $(R=5 \mathrm{~m}) \mathrm{Si}(111)$ crystal, computed for various source distances using a grid element size of $0.75 \mu \mathrm{m}$. The source distance $p=1.648 \mathrm{~m}$ is the position on the Rowland circle, $p=R \sin \theta_{\mathrm{B}}$. The curves are shifted on the $x$ axis for clarity. The reference curve is the same as in Fig. 5.

(Taking into account the refraction correction, the Darwin range is $4.21<\Delta \theta<14.03^{\prime \prime}$, or $20.4<\Delta \theta<68.0 \mu \mathrm{rad}$.)

When the distance to the source is changed, we observe that the RCs broaden. This is expected, as the incidence angle is not the same at every point on the crystal surface if the source is taken off the Rowland circle, and thus the diffraction condition is not fulfilled on the whole incident wavefront.

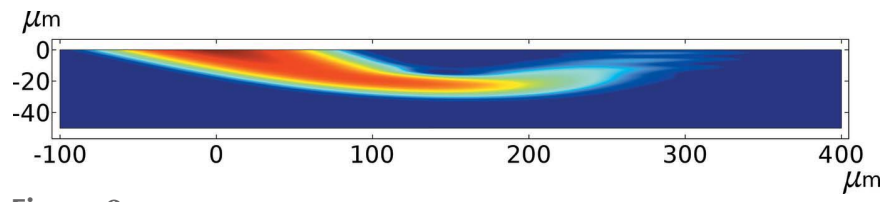

Figure 8

The total intensity inside the crystal at $\Delta \theta=1.31^{\prime \prime}(6.37 \mu \mathrm{rad})$, illustrating the mirage effect. A logarithmic scale is used for the colour mapping for visual clarity.

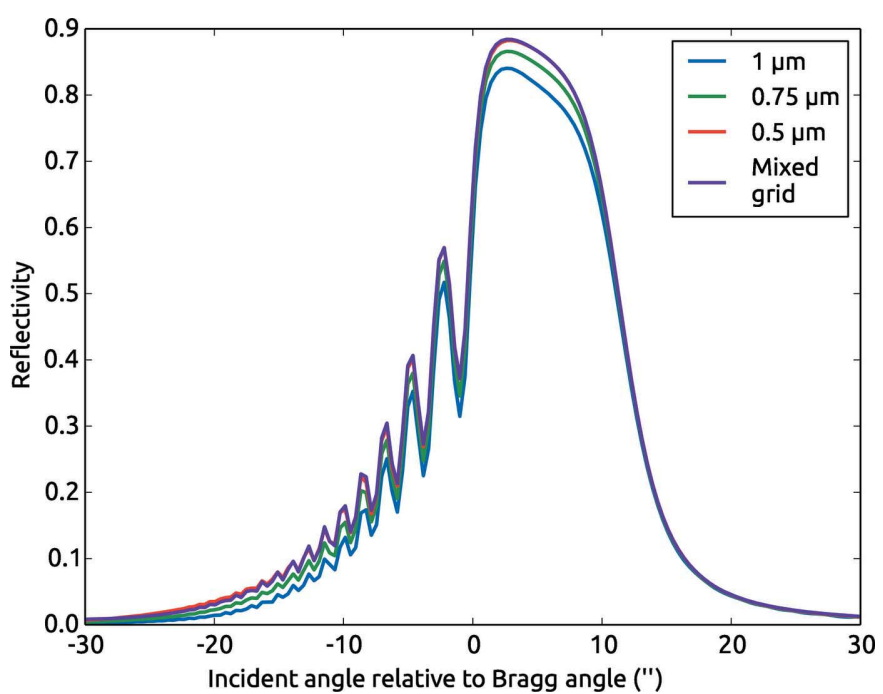

Figure 9

The reflectivity curves of the cylindrically bent $(R=0.5 \mathrm{~m}) \mathrm{Si}(111)$ crystal, computed for various mesh grid element sizes. The source was on the Rowland circle $\left(R \sin \theta_{\mathrm{B}}=0.165 \mathrm{~m}\right)$ and the FWHM of the Gaussian window was $100 \mu \mathrm{m}$.
The method was also tested for a smaller bending radius. To reflect the contemporary state of bent crystal analyser technology (Rovezzi et al., 2017), $R=0.5 \mathrm{~m}$ was chosen. As for the unbent and $5 \mathrm{~m}$ bent cases, the method was found to be stable, even when the shape of the RC is considerably affected by the deformation field. However, the convergence was found to be slower than previously observed, requiring grid element sizes of $0.5 \mu \mathrm{m}$ or even smaller. It turns out that this requirement for an increased grid density arises mainly from the stronger deformation in the $\mathbf{y}$ direction. By taking advantage of the freedom in the mesh construction in the FEM scheme, we scaled the distance of the elements by a factor of 0.5 in the $\mathbf{y}$ direction, leading to a grid with mixed element dimensions of $0.5 \times 1.0 \mu \mathrm{m}$ in the vertical and horizontal directions, respectively. The resulting curve was found to be the same as that with a uniform $0.5 \times 0.5 \mu \mathrm{m}$ mesh but with half the

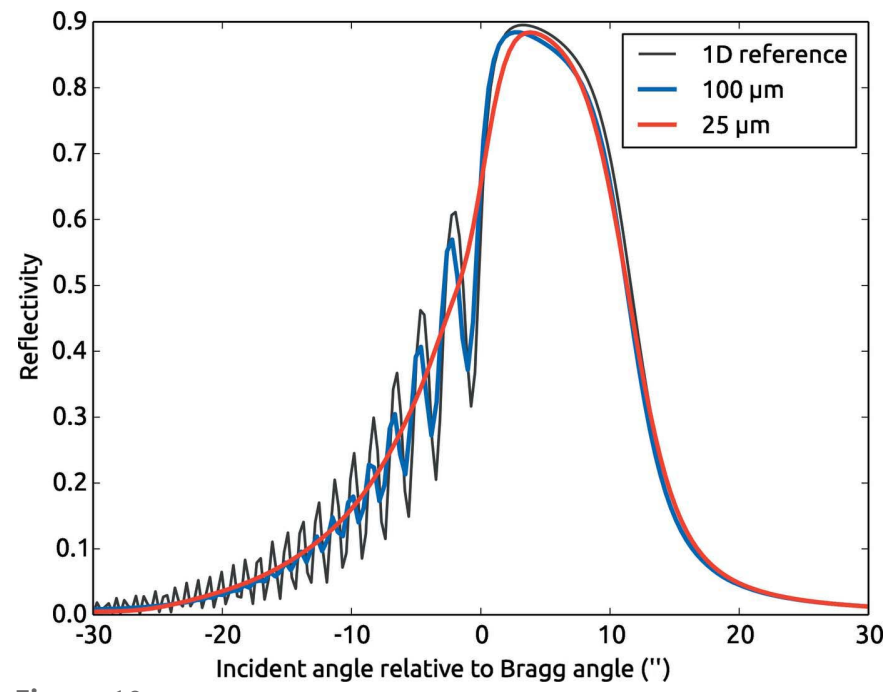

Figure 10

The reflectivity curves of the cylindrically bent $(R=0.5 \mathrm{~m}) \mathrm{Si}(111)$ crystal, computed for two differently sized Gaussian windows $(25$ and $100 \mu \mathrm{m})$. The source is on the Rowland circle $\left(R \sin \theta_{\mathrm{B}}=0.165 \mathrm{~m}\right)$. The reference curve in black is the solution to the one-dimensional form of the TakagiTaupin equations. 
number of grid elements, which again echoes the benefits of adaptive grid-construction schemes for speed and memory optimization. The RCs computed with different grids are presented in Fig. 9.

In order to validate the method for $R=0.5 \mathrm{~m}$, we computed the RC with a one-dimensional Takagi-Taupin solver (a slightly modified version of https:/github.com/aripekka/ pytakagitaupin) and compared it with the mixed-grid solutions. As shown in Fig. 10, the FEM results follow the general features of the reference curve but do not reproduce exactly the same details. This is to be expected, as the two-dimensional situation allows for the lateral dependence in the TTE that is missing in the one-dimensional case. This can be demonstrated by varying the footprint of the incident beam. For a sufficiently small beam, the overlap between the incident and diffracted waves is smaller, meaning that they interfere less with each other and thus lead to suppression of Pendellösung oscillations. Fig. 10 is a good example of how the one-

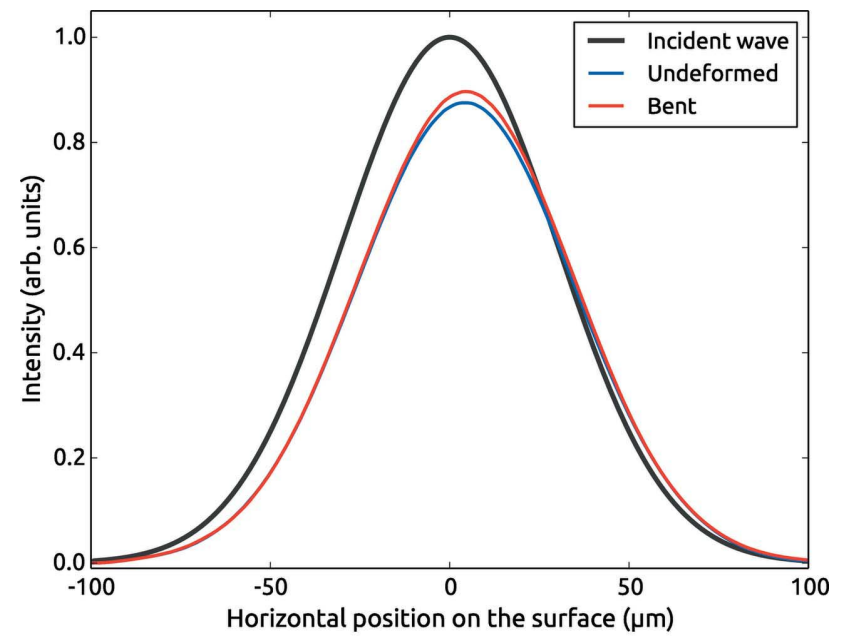

(a)

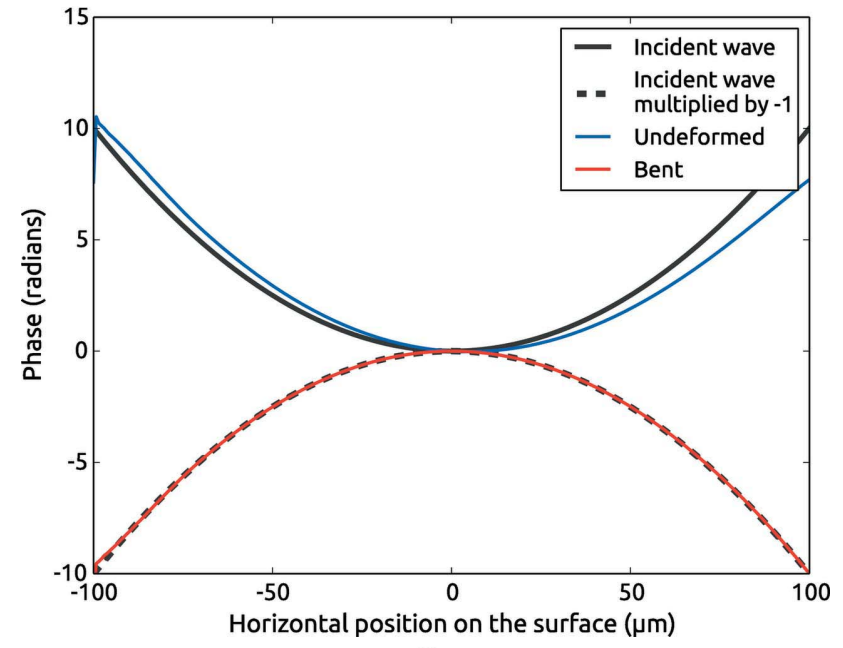

(b)

Figure 11

(a) The intensity distributions of the incident and diffracted waves on the crystal surface for the $5 \mathrm{~m}$ cylindrically bent crystal and the undeformed crystal (FWQM of the incident curve is $100 \mu \mathrm{m}$ ). The rocking angle was $\Delta \theta=6.9697^{\prime \prime}(33.79 \mu \mathrm{rad})$. The point source was on the Rowland circle $\left(R \sin \theta_{\mathrm{B}}=1.648 \mathrm{~m}\right)$. (b) The phases of the same waves. dimensional TTE are inherently different from the true twodimensional solution.

\subsection{Propagation and focusing of the diffracted beam}

We examine the propagation of the diffracted wave by the $5 \mathrm{~m}$ cylindrically bent crystal from $§ 7.2$. A grid element size of $0.75 \mu \mathrm{m}$ was used. The diffracted intensity and the phase of the diffracted wave [without the plane wave factor $\exp \left(i \mathbf{k}_{h} \cdot \mathbf{r}\right)$ ] on the crystal surface are presented in Fig. 11 for the top of the diffraction curve at $\Delta \theta=6.9697^{\prime \prime}(33.79 \mu \mathrm{rad})$. In addition to the bent crystal, the curves for a similar but undeformed crystal are shown for comparison.

The intensity distribution is found to be similar for both bent and undeformed crystals. This is not surprising, as the reflectivity curve is fairly unaffected by the deformation field. However, the phases of the diffracted waves differ drastically, owing mainly to the deformation phase factor $\exp (-i \mathbf{h} \cdot \mathbf{u})$. One should expect such a difference, as the proper focusing in

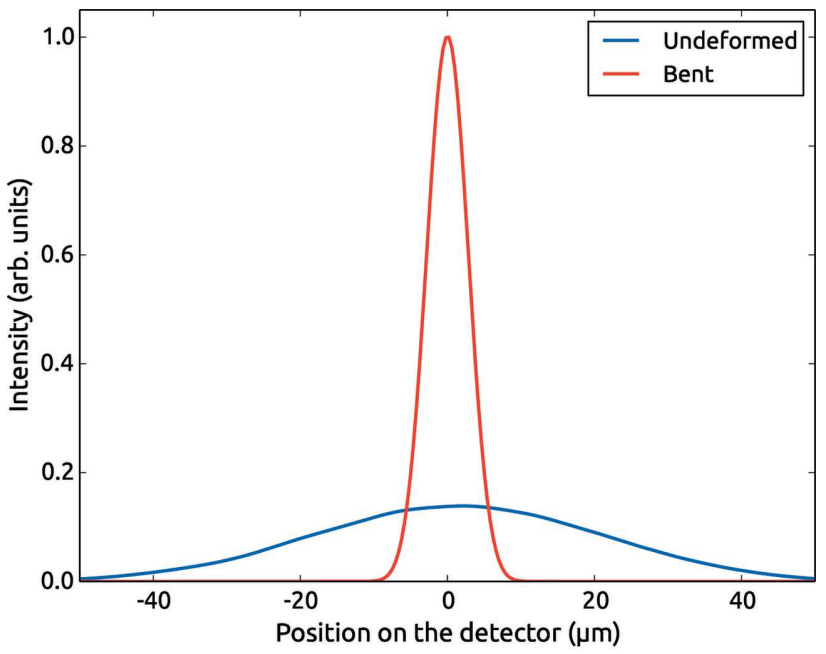

(a)

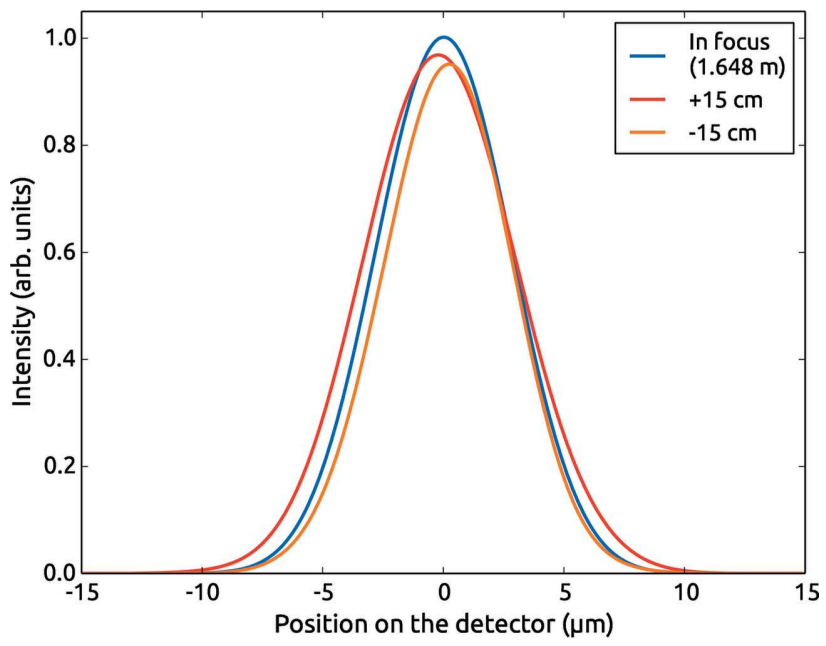

(b)

Figure 12

(a) The computed intensity distribution at the focal position on the Rowland circle. (b) The effect of the displacement of the detector through the focal spot of the cylindrical crystal. 
the Rowland-circle geometry is dependent on the correct bending of the crystal. The phase of the wave diffracted by the bent crystal coincides with the phase of the incident wave multiplied by -1 , which indicates that it describes a spherical wave with the same focal distance as the incident wave but propagating to the focal point instead of from it.

The proper focusing is confirmed by computing the Fresnel integral of equation (23) over the crystal surfaces. The intensities of the propagated waves on the Rowland circle are presented in Fig. 12. We indeed observe that the bent crystal focuses the beam, whereas for the unbent one the diffracted wave diverges. It is also confirmed that the optimal focal position of the cylindrical crystal is situated on the Rowland circle, as the peak intensity of the focal point decreases when the distance of the detector plane is altered.

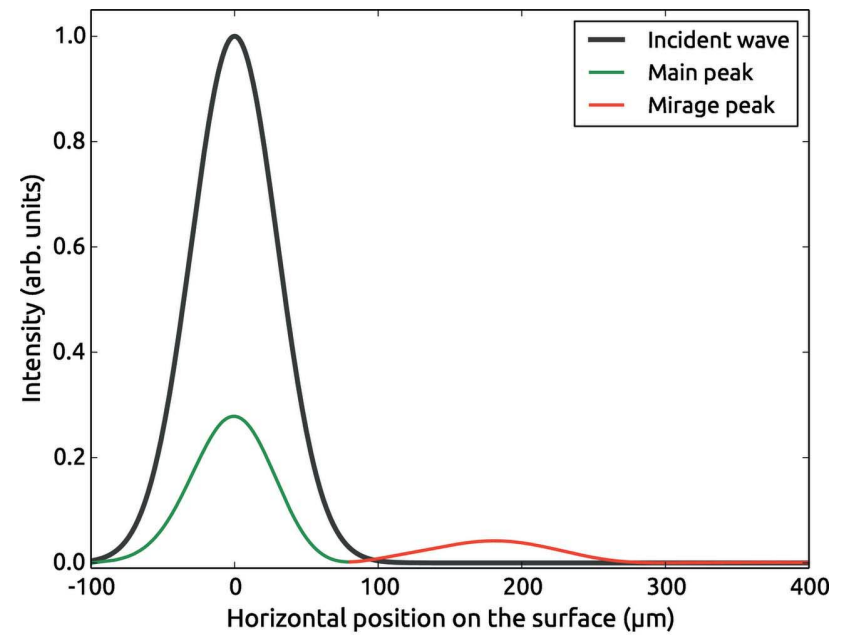

(a)

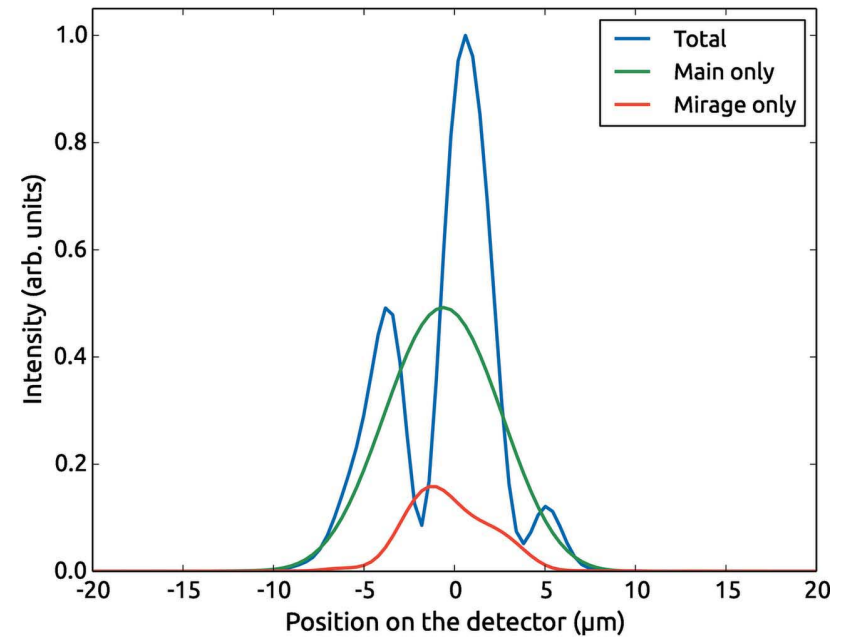

(b)

Figure 13

(a) The intensity distribution of the incident and diffracted waves on the crystal surface for the $5 \mathrm{~m}$ cylindrically bent crystal, showing the mirage effect (the FWQM of the incident curve is $100 \mu \mathrm{m}$ ). The rocking angle is $\Delta \theta=2.9293^{\prime \prime}(14.20 \mu \mathrm{rad})$. The point source is on the Rowland circle $\left(R \sin \theta_{\mathrm{B}}=1.648 \mathrm{~m}\right) .(b)$ Propagated intensities at the detector plane on the Rowland circle. The structure in the total intensity arises from the phase differences between the main and mirage peaks.
The behaviour of the focus in the presence of the mirage peak was also investigated. Fig. 13 shows the intensities on the crystal surface and on the detector plane at the Rowland circle for $\Delta \theta=2.9293^{\prime \prime}(14.20 \mu \mathrm{rad})$. In addition to computing the Fresnel integral over the whole surface, we divided the diffracted wave into the main peak and the mirage peak at $x=$ $80 \mu \mathrm{rad}$ and propagated the peaks separately to the detector plane. As can be seen in Fig. 13(a), the peaks focus nicely on the Rowland circle when propagated separately, with the mirage peak showing a slight shoulder on the right-hand side. However, when propagated together (Fig. 13b), they form a three-peaked structure in the intensity distribution. This interference-related phenomenon is caused by the phase differences between the main peak and the mirage peak which arise at different depths in the crystal.

Finally, the focal length for various source distances was studied. According to the lens equation (Chukhovskii \& Krisch, 1992), the source distance $p$ and the focal length $q$ are related in the Bragg symmetric case by

$$
\frac{1}{p}+\frac{1}{q}=\frac{2}{R \sin \theta_{\mathrm{B}}} .
$$

The validity of this relationship was investigated by computing the profiles of the propagated waves as a function of the detector plane distance for three different source distances. The peak intensities of the profiles were obtained and are plotted, together with the predictions of equation (34), in Fig. 14. The maxima of the peak intensities are found to be in good accordance with the lens equation, which is the expected result.

\section{Application to experiments}

For the sake of the generality of our approach, all calculations in the previous sections were performed under ideal or

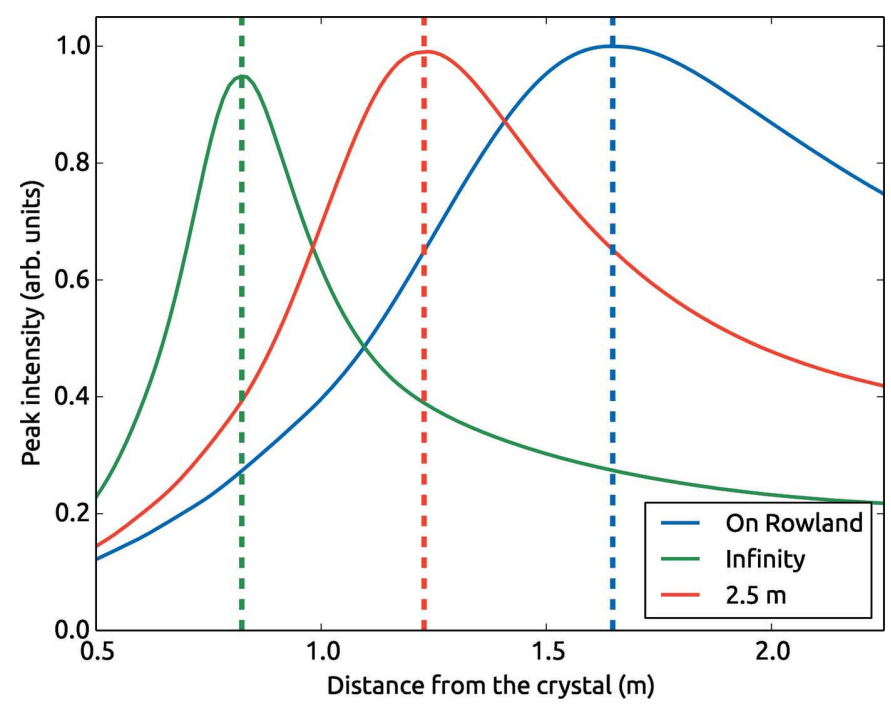

Figure 14

The peak intensities of the propagated wavefields as a function of distance of the detector plane for various source distances. The dashed vertical lines correspond to the focal lengths calculated using equation (34). 


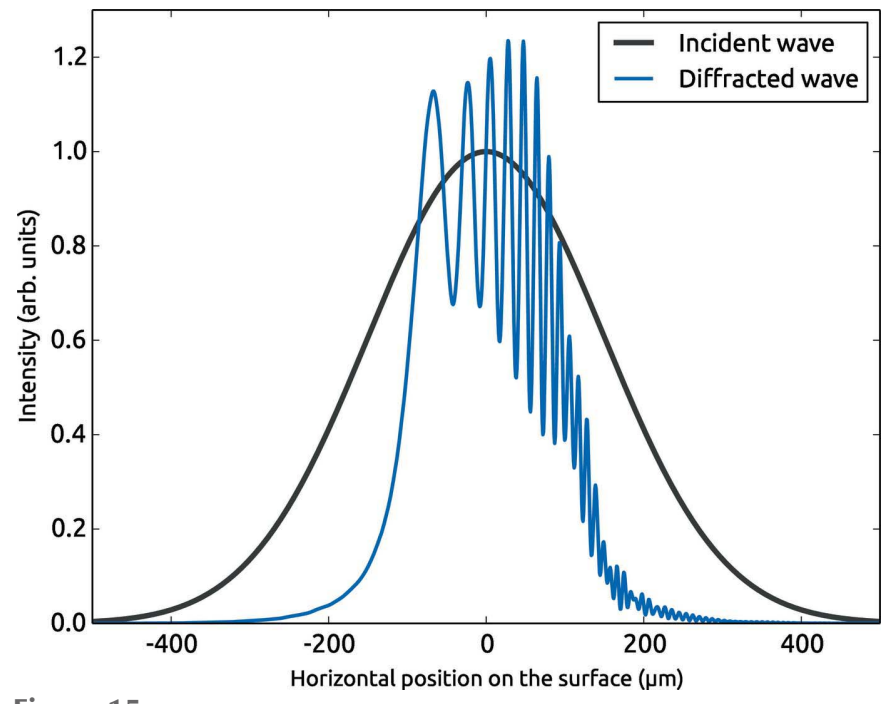

Figure 15

Computed intensities of the incident and diffracted waves on the crystal surface for simulation parameters chosen to match the experimental conditions. The illuminated crystal surface is $1000 \mu \mathrm{m}$ wide and the FWQM of the incident intensity profile is $500 \mu \mathrm{m}$. The width of the diffracted wave profile is roughly $200 \mu \mathrm{m}$.

simplified conditions, e.g. a monochromatic beam, no thermal load effects, perfectly cylindrical curvature, no mounting inaccuracies and a limited size of the incoming beam footprint on the crystal surface. The investigation of all these effects would depend strongly on each individual beamline optical setup to be examined and this would be far beyond the scope of the present research. However, the results of our simulations can be effectively used, for example, to predict the lowest size limit of the focal spot produced by a bent crystal in a given optical arrangement.

Bearing in mind this point of view, we considered the example of a knife-edge scanning measurement carried out on the dispersive EXAFS beamline ID24 (Hagelstein et al., 1995) at the ESRF, Grenoble, France. The aim of the experiment was to determine the focus width using the $\mathrm{Si}(111)$ reflection of a symmetrically cut curved polychromator at a mean photon energy $E=7 \mathrm{keV}$. The source was a secondary source (just downstream from a demagnification mirror), the size of which was evaluated to be between 40 and $45 \mu \mathrm{m}$. The distance to the curved polychromator was about $p=30 \mathrm{~m}$ and the focal distance was found to be $q=0.75 \mathrm{~m}$. The radius of curvature was estimated to be approximately $R=5.3 \mathrm{~m}$. The illuminated region on the crystal was about $15 \mathrm{~cm}$.

Following the simulation schemes described in the previous sections (taking a point source) and using the same parameters as in the ID24 experiment, we first checked whether our simulations would be able to determine a focal distance matching the experimental one. Crystal dimensions of $1000 \mu \mathrm{m}(\mathrm{H}) \times 50 \mu \mathrm{m}(\mathrm{V})$ were used. The FWQM of the incident intensity profile is $500 \mu \mathrm{m}$. The incidence angle was chosen to be in the centre of the rocking curve $\left(\Delta \theta=7.65^{\prime \prime}=\right.$ $37.1 \mu \mathrm{rad}$ ) Since the source is not on the Rowland circle, much larger areas would not lead to a better result as the diffraction condition is not fulfilled further away from the crystal centre.

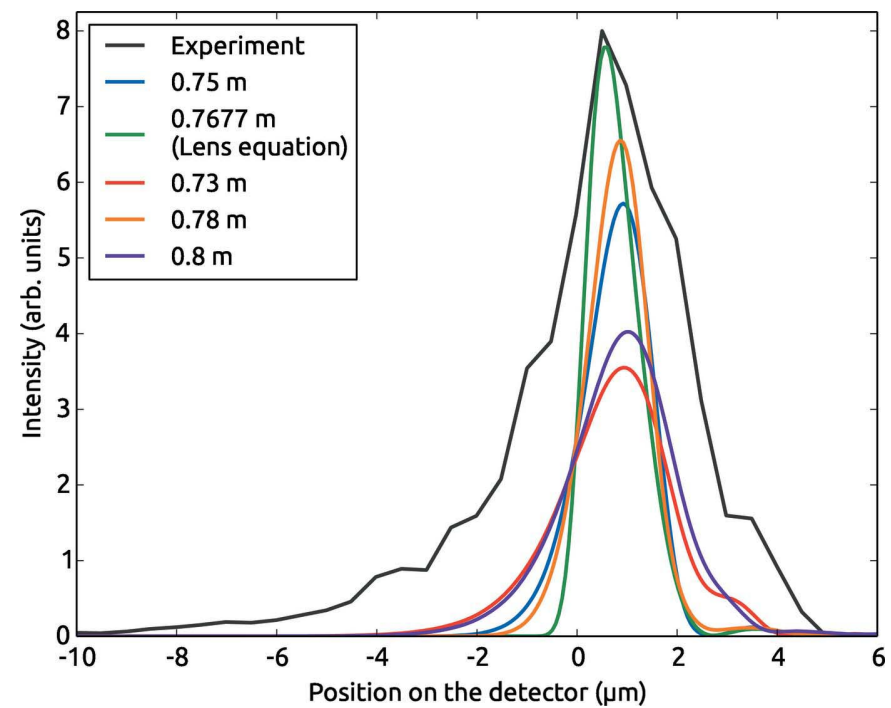

Figure 16

Simulated focal profiles at various focal distances compared with the experimental focus.

The simulated intensity profiles are presented in Fig. 15. The oscillations in the diffracted profile are due to interference between the incident and diffracted waves, which becomes prominent when the source is not on the Rowland circle and the illuminated area is large enough.

The correctness of the prediction is demonstrated in Fig. 16, showing focal spot distributions evaluated at different distances: the optimal focal distance is found to be $0.7677 \mathrm{~m}$, in accordance with the lens equation. The experimentally found value of $q=0.75 \mathrm{~m}$ is not too far away from the simulations when taking into account that the FWHM of the focus does not vary significantly in the range of $\pm 0.02 \mathrm{~m}$ around the optimum.

The calculated profile, as anticipated, is significantly narrower (Fig. 16): one can state that $\mathrm{FWHM}_{\exp }=2.8 \mu \mathrm{m}$, whereas $\mathrm{FWHM}_{\text {calc }}=1.4 \mu \mathrm{m}$. The latter value could be thought of by the experimenter as an ideal target to assess the level of mounting accuracy attained in the setup. The small side peak on the left of the central one (Fig. 16) might be the signature of the intensity spread owing to the Johann error (Wang et al., 2010).

\section{Conclusions and outlook}

A general approach to numerical solution of the TakagiTaupin equations in their integral representation using the finite-element framework, as implemented in the COMSOL Multiphysics package, has been presented. This provides a wide flexibility in the numerical solution of dynamical diffraction problems for both perfect and deformed crystals of arbitrary shape. The method is computationally efficient and comparable to the classical, albeit less flexible and powerful, finite-difference approach in conventional cases of simplistic geometries, i.e. two-dimensional Cartesian systems with mostly straight-line boundaries. In particular, we have shown the versatility of the FEM computational framework in 
elucidating a wealth of noteworthy, yet hitherto not fully explained, aspects of the focusing behaviour of cylindrically bent crystals in symmetric Bragg geometry.

The impact of our approach is meant to go far beyond the limited scope of the present work in order to encompass a vast class of numerical problems related to solving not only twodimensional but also three-dimensional X-ray and neutron dynamical diffraction problems in the Bragg and Laue geometries. For instance, the FEM method may be applied to investigating numerically the possibility of obtaining rocking curves of the Darwin type in Bragg geometry, instead of the Ewald type, by using crystal plates featuring grooves on their back surface, as suggested by Freund \& Rehm (2014). Since the FEM is one of the most powerful methods known to successfully address boundary and/or initial value problems described by PDEs, and since the COMSOL Multiphysics package allows easy integration of user-defined equation systems into its kernel and prompt display of the results thanks to its built-in graphical facilities, we hope to disclose and encourage the application of this more general technique among the relevant scientific community. For this purpose, we have provided an open-access location for our COMSOL files. They can be freely downloaded from https:/github.com/ aripekka/fem-takagi-taupin.

\section{APPENDIX $A$}

\section{Derivatives in oblique and Cartesian coordinate} systems

Consider an arbitrary function $F=F(x, y)$. In terms of the Cartesian coordinate basis $\left\{\mathbf{e}_{x}, \mathbf{e}_{y}\right\}$, the unit vectors, we define an oblique system $\left(s_{0}, s_{h}\right)$ with the base vectors

$$
\mathbf{s}_{0}=\cos \alpha \mathbf{e}_{x}-\sin \alpha \mathbf{e}_{y}, \quad \mathbf{s}_{h}=\cos \alpha^{\prime} \mathbf{e}_{x}+\sin \alpha^{\prime} \mathbf{e}_{y} .
$$

Thus

$$
\begin{aligned}
s_{0} \cdot \nabla F & =\left(\cos \alpha \mathbf{e}_{x}-\sin \alpha \mathbf{e}_{y}\right)\left(\mathbf{e}_{x} \frac{\partial F}{\partial x}+\mathbf{e}_{y} \frac{\partial F}{\partial y}\right) \\
& =\cos \alpha \frac{\partial F}{\partial x}-\sin \alpha \frac{\partial F}{\partial y} .
\end{aligned}
$$

Also

$$
\begin{aligned}
\nabla \cdot\left(s_{0} F\right) & =\left(\mathbf{e}_{x} \frac{\partial}{\partial x}+\mathbf{e}_{y} \frac{\partial}{\partial y}\right)\left(F \cos \alpha \mathbf{e}_{x}-F \sin \alpha \mathbf{e}_{y}\right) \\
& =\cos \alpha \frac{\partial F}{\partial x}-\sin \alpha \frac{\partial F}{\partial y} .
\end{aligned}
$$

Switching to the oblique system, we can write $F\left(s_{0}, s_{h}\right)=$ $F\left[x\left(s_{0}, s_{h}\right), y\left(s_{0}, s_{h}\right)\right]$. Since $x=\left(s_{0} \cos \alpha+s_{h} \cos \alpha^{\prime}\right)$ and $y=$ $\left(-s_{0} \sin \alpha+s_{h} \sin \alpha^{\prime}\right)$,

$$
\frac{\partial F}{\partial s_{0}}=\frac{\partial x}{\partial s_{0}} \frac{\partial F}{\partial x}+\frac{\partial y}{\partial s_{0}} \frac{\partial F}{\partial y}=\cos \alpha \frac{\partial F}{\partial x}-\sin \alpha \frac{\partial F}{\partial y} .
$$

Pulling all together, we thus obtain a useful identity

$$
\mathbf{s}_{0} \cdot \nabla F=\nabla \cdot\left(\mathbf{s}_{0} F\right)=\frac{\partial F}{\partial s_{0}} .
$$

Analogously for $s_{h}$

$$
\mathbf{s}_{h} \cdot \nabla F=\nabla \cdot\left(\mathbf{s}_{h} F\right)=\frac{\partial F}{\partial s_{h}} .
$$

\section{APPENDIX $B$}

\section{Deformation field of a cylindrically bent crystal}

According to the conventional elastic theory of thin crystal plates (Nesterets \& Wilkins, 2006), the deformation field $\left(\mathbf{u}_{x}, \mathbf{u}_{y}\right)$ for a cylindrically bent isotropic crystal of thickness $t$ is given by

$$
u_{x}=-\frac{x}{R}\left(y+\frac{t}{2}\right), \quad u_{y}=\frac{1}{2 R}\left[x^{2}+v\left(y+\frac{t}{2}\right)^{2}\right],
$$

where $R$ is the bending radius and $v$ is the Poisson ratio. The top surface of the crystal before deformation is assumed to be at $y=0$ and the bottom at $y=-t$.

In the symmetric Bragg case, one obtains

$$
\mathbf{h} \cdot \mathbf{u}=\frac{h}{2 R}\left[x^{2}+v\left(y+\frac{t}{2}\right)^{2}\right]=\frac{\pi}{R d}\left[x^{2}+v\left(y+\frac{t}{2}\right)^{2}\right],
$$

where $d$ is the interplanar distance of the considered Bragg reflection. Thus, the deformation term $\partial_{h}(\mathbf{h} \cdot \mathbf{u})$ becomes

$$
\begin{aligned}
\frac{\partial_{h}(\mathbf{h} \cdot \mathbf{u})}{\partial s_{h}} & =\frac{\partial(\mathbf{h} \cdot \mathbf{u})}{\partial x} \cos \theta_{\mathrm{B}}+\frac{\partial(\mathbf{h} \cdot \mathbf{u})}{\partial y} \sin \theta_{\mathrm{B}} \\
& =\frac{2 \pi}{R d}\left[x \cos \theta_{\mathrm{B}}+v\left(y+\frac{t}{2}\right) \sin \theta_{\mathrm{B}}\right] .
\end{aligned}
$$

Following Gronkowski (1991), the deformation is considered weak for the symmetric Bragg case if

$$
\left|\frac{\partial^{2}(\mathbf{h} \cdot \mathbf{u})}{\partial s_{0} \partial s_{h}}\right| \ll \frac{\pi^{2} C^{2}\left|\chi_{h} \chi_{\bar{h}}\right|}{\lambda^{2} \tan \theta_{\mathrm{B}}} .
$$

Since

$$
\frac{\partial^{2}(\mathbf{h} \cdot \mathbf{u})}{\partial s_{0} \partial s_{h}}=\frac{2 \pi}{R d}\left(\cos ^{2} \theta_{\mathrm{B}}-v \sin ^{2} \theta_{\mathrm{B}}\right),
$$

the condition (44) takes the form

$$
R \gg \frac{8 d \sin ^{2} \theta_{\mathrm{B}} \tan \theta_{\mathrm{B}}}{\pi C^{2}\left|\chi_{h} \chi_{\bar{h}}\right|}\left|\cos ^{2} \theta_{\mathrm{B}}-v \sin ^{2} \theta_{\mathrm{B}}\right| .
$$

\section{Acknowledgements}

We are greatly indebted to Olivier Mathon (ESRF, Grenoble) for the provision of the experimental data reported in the text. Computing resources were provided by the CSC-IT Centre for Science, Finland.

\section{Funding information}

Funding for this research was provided by Academy of Finland (grant No. 1295696 to Ari-Pekka Honkanen) and the doctoral programme in Materials Research and Nanosciences 
(MATRENA) at the University of Helsinki (to Ari-Pekka Honkanen).

\section{References}

Apolloni, A., Mana, G., Palmisano, C. \& Zosi, G. (2008). Acta Cryst. A64, 549-559.

Authier, A. (2004). Dynamical Theory of X-ray Diffraction. Oxford University Press.

Authier, A., Malgrange, C. \& Tournarie, M. (1968). Acta Cryst. A24, 126-136.

Balibar, F. \& Authier, A. (1967). Phys. Status Solidi B, 21, 413-422.

Carvalho, C. A. M. \& Epelboin, Y. (1993a). Acta Cryst. A49, 460-467.

Carvalho, C. A. M. \& Epelboin, Y. (1993b). Acta Cryst. A49, 467-473.

Chukhovskii, F. N., Chang, W. Z. \& Förster, E. (1994). J. Appl. Cryst. 27, 971-979.

Chukhovskii, F. N., Gabrielyan, K. T. \& Petrashen', P. V. (1978). Acta Cryst. A34, 610-621.

Chukhovskii, F. N. \& Krisch, M. (1992). J. Appl. Cryst. 25, 211-213.

Epelboin, Y. (1985). Mater. Sci. Eng. 73, 1-43.

Epelboin, Y. \& Riglet, P. (1979). Phys. Status Solidi A, 54, 547-556.

Freund, A. \& Rehm, C. (2014). J. Phys. Conf. Ser. 528, 012002.

Gronkowski, J. (1991). Phys. Rep. 206, 1-41.

Gronkowski, J. \& Malgrange, C. (1984). Acta Cryst. A40, 507-514.

Guigay, J. P. \& Ferrero, C. (2016). Acta Cryst. A72, 489-499.

Guigay, J. P., Morawe, C., Mocella, V. \& Ferrero, C. (2008). Opt. Express, 16, 12050-12059.

Hagelstein, M., Ferrero, C., Hatje, U., Ressler, T. \& Metz, W. (1995). J. Synchrotron Rad. 2, 174-180.

Honkanen, A.-P., Ferrero, C., Guigay, J. \& Mocella, V. (2017). Proc. SPIE, 10236, 1023605.

Honkanen, A.-P., Monaco, G. \& Huotari, S. (2016). J. Appl. Cryst. 49, 1284-1289.

Honkanen, A.-P., Verbeni, R., Simonelli, L., Moretti Sala, M., Monaco, G. \& Huotari, S. (2014). J. Synchrotron Rad. 21, 104-110.

Katagawa, T. \& Kato, N. (1974). Acta Cryst. A30, 830-836.

Lagomarsino, S., Cedola, A., Di Fonzo, S., Jark, W., Mocella, V., Pelka, J. B. \& Riekel, C. (2002). Cryst. Res. Technol. 37, 758-769.

Litzman, O. \& Janáček, Z. (1974). Phys. Status Solidi A, 25, 663-666.
Mocella, V., Ferrero, C. \& Guigay, J. (2015). Proc. SPIE, 9510, $95100 \mathrm{H}$.

Mocella, V., Ferrero, C., Hrdý, J., Wright, J., Pascarelli, S. \& Hoszowska, J. (2008). J. Appl. Cryst. 41, 695-700.

Mocella, V., Guigay, J. P., Hrdý, J., Ferrero, C. \& Hoszowska, J. (2004). J. Appl. Cryst. 37, 941-946.

Mocella, V., Lee, W.-K., Tajiri, G., Mills, D., Ferrero, C. \& Epelboin, Y. (2003). J. Appl. Cryst. 36, 129-136.

Morawe, C., Guigay, J., Mocella, V. \& Ferrero, C. (2008). Opt. Express, 16, 16138-16150.

Nesterets, Y. \& Wilkins, S. (2006). Radiat. Phys. Chem. 75, 1981-1985.

Nesterets, Y. I. \& Wilkins, S. W. (2008). J. Appl. Cryst. 41, 237-248.

Oden, J. T. \& Reddy, J. N. (2012). An Introduction to the Mathematical Theory of the Finite Element Method. Mineola: Courier Corporation.

Osterhoff, M., Morawe, C., Ferrero, C. \& Guigay, J. (2013). Opt. Lett. 38, 5126-5129.

Penning, P. \& Polder, D. (1961). Philips Res. Rep. 16, 419-440.

Podorov, S., Renner, O., Wehrhan, O. \& Förster, E. (2001). J. Phys. D Appl. Phys. 34, 2363-2368.

Reddy, J. N. (2006). An Introduction to the Finite Element Method, 3rd ed., McGraw-Hill Series in Mechanical Engineering. New York: McGraw-Hill.

Rovezzi, M., Lapras, C., Manceau, A., Glatzel, P. \& Verbeni, R. (2017). Rev. Sci. Instrum. 88, 013108.

Sánchez del Río, M. \& Dejus, R. (2011). Proc. SPIE, 8141, 814115.

Sanchez del Rio, M., Perez-Bocanegra, N., Shi, X., Honkimäki, V. \& Zhang, L. (2015). J. Appl. Cryst. 48, 477-491.

Sutter, J., Amboage, M., Hayama, S. \& Díaz-Moreno, S. (2010). Nucl. Instrum. Methods Phys. Res. A, 621, 627-636.

Takagi, S. (1962). Acta Cryst. 15, 1311-1312.

Takagi, S. (1969). J. Phys. Soc. Jpn, 26, 1239-1253.

Taupin, D. (1964). Bull. Soc. Fr. Miner. Cristall. 87, 469-511.

Tolentino, H., Dartyge, E., Fontaine, A. \& Tourillon, G. (1988). J. Appl. Cryst. 21, 15-22.

Wang, E., Beiersdorfer, P., Gu, M., Bitter, M., Delgado-Aparicio, L., Hill, K. W., Reinke, M., Rice, J. E. \& Podpaly, Y. (2010). Rev. Sci. Instrum. 81, 10E329.

Yan, H. \& Li, L. (2014). Phys. Rev. B, 89, 014104. 